\title{
Cu-O network dependent core-hole screening in low-dimensional cuprates: A theoretical analysis
}

\author{
C. Waidacher, J. Richter, and K. W. Becker \\ Institut für Theoretische Physik, Technische Universität Dresden, D-01062 Dresden, Germany
}

\begin{abstract}
We analyze the influence of the dimensionality on the $\mathrm{Cu} 2 p_{3 / 2}$ core level X-ray photoemission spectra of undoped cuprates. Both exchange splitting and delocalization properties are described within one framework using a multi-band Hubbard model. The spectral intensity is calculated by means of the Mori-Zwanzig projection technique for a $\mathrm{CuO}_{4}$ plaquette ('zero'-dimensional), an infinite $\mathrm{CuO}_{3}$ chain (one-dimensional), and an infinite $\mathrm{CuO}_{2}$ plane (two-dimensional). The results are compared to the spectra of $\mathrm{Bi}_{2} \mathrm{CuO}_{4}, \mathrm{Sr}_{2} \mathrm{CuO}_{3}$, and $\mathrm{Sr}_{2} \mathrm{CuO}_{2} \mathrm{Cl}_{2}$. Our analysis allows to distinguish between effects of the $\mathrm{Cu}-\mathrm{O}$ geometry and effects from material-specific properties.
\end{abstract}

\section{INTRODUCTION}

The influence of dimensionality and lattice geometry on the electronic structure of crystals is a classical topic in solid state physics. However, in the presence of strong electronic correlations like those observed in the high$T_{c}$ superconducting cuprates, the theoretical analysis of electronic properties is especially difficult. Recently, the electronic structure of various undoped cuprates has been studied experimentally using core-level X-ray photoemission spectroscopy (XPS) 1. B B comparing these spectra to the results of theoretical model calculations we can try to improve our understanding of the electronic properties of the cuprates. In addition, a theoretical analysis allows us to check the validity of the microscopic models used for the calculations, and it provides values for the physical parameters which are contained in the model Hamiltonians.

The core-level photoemission process leads to a final state in which an electron is missing in the $2 p_{3 / 2}$ core orbital of a $\mathrm{Cu}$ site. The resulting positive charge is screened by valence electrons, predominantly from $\mathrm{Cu}$ $3 d_{x^{2}-y^{2}}$ and $\mathrm{O} 2 p_{x, y}$ orbitals. The contribution of these screening processes to the final state determines the detailed form of the experimental spectrum. Therefore, core-level spectra contain information about the dynamics of valence electrons.

Since the atomic ground state of the $\mathrm{Cu}-\mathrm{O}$ system is a $2 p^{6} 3 d^{9}$ configuration, it is advantageous to use the hole picture. In this case the core-level photoemission process amounts to the creation of a core hole which has a strong repulsive Coulomb interaction with the valence hole in the $3 d_{x^{2}-y^{2}}$ orbital of the same $\mathrm{Cu}$ site.

Experimentally, the $\mathrm{Cu} 2 p$ core-level spectrum of many formally divalent $\mathrm{Cu}$ compounds shows a dpminant main line and a pronounced satellite structure. 6 In analogy to an approach for metals,, this satellite structure was assigned to a ("poorly screened") final state (denoted $3 d^{9}$ ) in which the valence hole largely remains on the $\mathrm{Cu}$ site. 8 In this way, the structure of the satellite could be explaingd by multiplet splitting due to the remaining $d$-hole. 612

The main line was interpreted as originating from a final state in which the hole resides in the neighbour- ing ligands (denoted $3 d^{10} \underline{\mathrm{L}}$ ). However, for several compounds the main line is found to be asymmetric, and to have a large width. For instance, three substructures were identified in the main line of $\mathrm{Bi}_{2} \mathrm{Sr}_{2} \mathrm{CaCu}_{2} \mathrm{O}_{8} \mathrm{H} 3$ and $\mathrm{Sr}_{2} \mathrm{CuO}_{2} \mathrm{Cl}_{2}$ l. 2 (which will be analyzed in this paper). Substructures of this kind are the reason why the main line in the $\mathrm{Cu} 2 p_{3 / 2}$ spectra cannot be attributed to a local $3 d^{10} \underline{L}$ excitation only: This interpretation does not account for the asymmetry and the large width of the main line.

An alternative explanation was found using numerical exact diponalization for a chain of three plaquettes $\left(\mathrm{Cu}_{3} \mathrm{O}_{10}\right) .4 .5$ It was shown that the valence hole could delocalize further in the crystal. This leads to a lowest eigenstate of $3 d^{10}$ character, in which the hole is mainly pushed out onto the meighbouring $\mathrm{CuO}_{4}$ units, forming a Zhang-Rice singlet 16 These delocalization processes in the hole picture are equivalent to the screening processes in the electron picture.

Obviously, this screening of the care hole depends on the geometry of the $\mathrm{CuO}$ network 17 This suggests the possibility of finite-size effects in cluster calculations $18 \mathrm{In}$ fact, convergence of the spectra with respect to the system size was found only for chain clusters with lengths of seven plaquettes $\left(\mathrm{Cu}_{7} \mathrm{O}_{21}\right) .19$ These calculations led to a satisfactory agreement with the experimental results for $\mathrm{Sr}_{2} \mathrm{CuO}_{3}$. 19

Another popular model for the description of corelevel speftroscopy is the single-site Anderson impurity model.20 21 Recently, the influence of dimensionality on $\mathrm{Cu} 2 p_{3 / 2}$ spectra of several cuprates was analyzed using this model.22 Overall, very good agreement with the experimental main lines was obtained. The satellite structures, however, were not discussed. In particular, no multiplet effects were included. Furthermore, the absolute energetical positions of the main lines of $\mathrm{Sr}_{2} \mathrm{CuO}_{3}$ and $\mathrm{Sr}_{2} \mathrm{CuO}_{2} \mathrm{Cl}_{2}$ were not correctly reproduced. These shortcomings are avoided in our treatment.

In the present paper, we discuss the influence of of the dimensionality on the $\mathrm{Cu} 2 p_{3 / 2}$ core level spectra of undoped cuprates by means of the Mori-Zwanzig projection technique. Both exchange splitting and delocalization properties are described within one framework using a 
multi-band Hubbard model. In addition, a clear description of the final states is obtained.

Our analysis allows to distinguish between effects of the $\mathrm{Cu}-\mathrm{O}$ geometry and effects from material-specific properties. Examples for material-specific properties are the $\mathrm{Cu}-\mathrm{O}$ distance or components which do not belong to the $\mathrm{Cu}-\mathrm{O}$ network. Some of these properties are described by the values of model parameters like the $\mathrm{Cu}-\mathrm{O}$ charge-transfer energy or the $\mathrm{Cu}-\mathrm{O}$ hybridization strength. A phenomenological analysis cannot conclusively determine whether an effect is due to dimensionality or not. For instance, due to differences in materialspecific properties a peak which is brought about by a similar process in two different compounds may not appear at the same binding energy in both spectra. Therefore, the investigation of microscopic models is necessary.

We compare our calculations with the experimental spectra of three materials which consist of different $\mathrm{Cu}-\mathrm{O}$ networks: $\mathrm{Bi}_{2} \mathrm{CuO}_{4}$ which contains separated $\mathrm{CuO}_{4}$ plaquettes 23 ("zero"-dimensional $\mathrm{Cu}-\mathrm{O}_{4}$ network), $\mathrm{Sr}_{2} \mathrm{CuO}_{3}$ where the plaquettes form linear chains 24 (onedimensional $\mathrm{Cu}-\mathrm{O}_{3}$ network), and $\mathrm{Sr}_{2} \mathrm{CuO}_{2} \mathrm{Cl}_{2}$ which contains $\mathrm{CuO}_{2}$ planes 25 (two-dimensional $\mathrm{Cu}-\mathrm{O}_{2}$ network).

In the case of zero-dimensional edge-shared or separated plaquettes (like $\mathrm{Bi}_{2} \mathrm{CuO}_{4}$ ) the main line consists of a single feature which is explained by a local process in which the valence hole mpves from the core-hole site to the surrounding $\mathrm{O}$ sites 19,26 For one-dimensional zigzag or linear chains (like $\mathrm{Sr}_{2} \mathrm{CuO}_{3}$ ) two features contribute to the main line. In the case of two-dimensional planar systems (like $\mathrm{Sr}_{2} \mathrm{CuO}_{2} \mathrm{Cl}_{2}$ ) the main line is composed of at least three features. In addition to these effects there is an interesting trend in the intensity ratio $I_{s} / I_{m}$ between the satellite and the main line. For zero-dimensional systems this ratio is rather large, e.g. $I_{s} / I_{m} \leq 0.58$ for $\mathrm{Bi}_{2} \mathrm{CuO}_{4}$. On the other hand, in the case of one-dimensional networks the ratio is small, for instance $I_{s} / I_{m}=0.37$ for $\mathrm{Sr}_{2} \mathrm{CuO}_{3}$. The ratio for twodimensional systems lies in-between these values, e.g. $I_{s} / I_{m}=0.52$ for $\mathrm{Sr}_{2} \mathrm{CuO}_{2} \mathrm{Cl}_{2}$.

The paper is organized as follows. In Sec. II we introduce the model Hamiltonian and describe the method of calculation. Section III contains a discussion of the results. Our conclusions are summarized in Sec. IV.

\section{MODEL AND CALCULATION}

For the calculation of the $\mathrm{Cu} 2 \mathrm{p}_{3 / 2}$ photoemission spectra we use a multi-band Hubbard Hamiltonian to describe the $\mathrm{Cu}-\mathrm{O}$ network, while additional terms represent the interaction between the core hole and valence holes on the core-hole site. The full Hamiltonian in the hole picture is

$$
H=\Delta \sum_{j \sigma} n_{j \sigma}^{p}+U_{d d} \sum_{i} n_{i \uparrow}^{d} n_{i \downarrow}^{d}+U_{d c} \sum_{\sigma \xi} n_{0 \sigma}^{d} n_{0 \xi}^{c}
$$

$$
\begin{aligned}
& +I_{d c} \mathbf{S}_{0}^{d} \cdot \mathbf{J}_{0}^{c}+t_{p d} \sum_{\langle i j\rangle \sigma} \phi_{p d}^{i j}\left(p_{j \sigma}^{\dagger} d_{i \sigma}+h . c .\right) \\
& +t_{p p} \sum_{\left\langle j j^{\prime}\right\rangle \sigma} \phi_{p p}^{j j^{\prime}} p_{j \sigma}^{\dagger} p_{j^{\prime} \sigma}
\end{aligned}
$$

where $d_{i \sigma}^{\dagger}\left(p_{j \sigma}^{\dagger}\right)$ create a hole with spin $\sigma$ in the $i$-th $\mathrm{Cu}$ $3 d$ orbital ( $j$-th O $2 p$ orbital) and $n_{i \sigma}^{d}\left(n_{j \sigma}^{p}\right)$ are the corresponding occupation-number operators. The first and second term on the r.h.s. of Eq. (11) describe the chargetransfer energy $\Delta$ and the on-site Coulomb repulsion $U_{d d}$ between $\mathrm{Cu} 3 d$ valence holes. The third and fourth term represent the local Coulomb repulsion $U_{d c}$ and the effective exchange interaction $I_{d c}$ between the $3 d$ valence holes and the $2 p_{3 / 2}$ core hole at the core-hole site (which is taken to be site $i=0)$. $\mathbf{S}_{0}^{d}$ is the spin- $1 / 2$ operator of a $\mathrm{Cu} 3 d$ hole, while $\mathbf{J}_{0}^{c}$ and $n_{0 \xi}^{c}$ are the pseudo-spin $3 / 2$ operator and the number operator of the $2 p_{3 / 2}$ core hole (with $\xi= \pm 3 / 2, \pm 1 / 2$ ) at site $i=0$. Finally, the last two terms on the r.h.s. of Eq. (11) describe the hybridization of $\mathrm{Cu} 3 d$ and $\mathrm{O} 2 p$ orbitals (hopping strength $t_{p d}$ ) and of $\mathrm{O} 2 p$ orbitals (hopping strength $t_{p p}$ ). The factors $\phi_{p d}^{i j}$ and $\phi_{p p}^{j j^{\prime}}$ give the correct sign for the hopping processes and $\langle i j\rangle$ denotes the summation over nearest neighbor pairs. Hamiltonian (11) describes delocalization processes and multiplet splitting within one framework.

The spectral intensity $I(\omega)$, as a function of binding energy $\omega$, is obtained from the hole-hole correlation function

$$
\begin{gathered}
I(\omega)=-\sum_{\xi} \Im\left[G_{00}^{\xi}(\omega+i 0)\right], \\
G_{00}^{\xi}(\omega+i 0)=\left\langle\Psi\left|c_{0 \xi} \frac{1}{\omega+i 0-\mathcal{L}} c_{0 \xi}^{\dagger}\right| \Psi\right\rangle,
\end{gathered}
$$

where $c_{0 \xi}^{\dagger}$ creates a core hole with pseudo-spin $\xi$ at site $i=0$. $\mathcal{L}$ is the Liouville operator defined by $\mathcal{L} A=[H, A]$ for any operator $A .|\Psi\rangle$ is the full ground state of $H$ before the core hole is created in the photoemission process.

As illustrated in Ref. 27, the full ground state $|\Psi\rangle$ can be constructed by starting from the atomic, Néel-ordered ground state $\left|\psi_{0}\right\rangle$. In $\left|\psi_{0}\right\rangle$ all $\mathrm{O}$ sites are empty and every $\mathrm{Cu}$ site is singly occupied with alternating spin direction. Using an exponential transformation, $\left|\psi_{0}\right\rangle$ is approximately transformed into the full ground state $|\Psi\rangle$

$$
|\Psi\rangle=\exp \left(\sum_{i \alpha} \lambda_{\alpha} F_{i, \alpha}\right)\left|\Psi_{N}\right\rangle
$$

with fluctuation operators $F_{i, \alpha}$ and fluctuation strengths $\lambda_{\alpha}$. The operators $F_{i, \alpha}$ describe delocalizations of a valence hole which was initially located at $\mathrm{Cu}$ site $i$. For instance, fluctuation $F_{i, 2 d}$ describes the creation of doubly occupied $\mathrm{Cu}$ sites due to the hopping of a hole from $\mathrm{Cu}$ site $i$, via nearest neighbor $\mathrm{O}$ site $j$, to the nearest neighbor $\mathrm{Cu}$ sites $k$ 


$$
F_{i, 2 d}=\sum_{j k \sigma} n_{k \bar{\sigma}}^{d} d_{k \sigma}^{\dagger}\left(1-n_{j \sigma}^{p}\right) d_{i \sigma} .
$$

In the case of a single $\mathrm{CuO}_{4}$ plaquette one fluctuation operator is sufficient to obtain the exact ground state. For a $\mathrm{CuO}_{3}$ chain we use up to nine fluctuation operators thus allowing for delocalizations leading as far as to the next-nearest neighbor plaquette. Due to its higher symmetry, the ground state of a $\mathrm{CuO}_{2}$ plane is well described by only five fluctuation operators. For a detailed description of the fluctuation operators $F_{i, \alpha}$ see Ref. 27 . The fluctuation strengths $\lambda_{\alpha}$ are determined using the set of equations

$$
0=\left\langle\Psi\left|\left[H, F_{\alpha}^{\dagger}\right]\right| \Psi\right\rangle, \alpha=1,2, \ldots .
$$

which follows from the condition that $|\Psi\rangle$ is an eigenstate of the full Hamiltonian (11). The parameters $\lambda_{\alpha}$ are found to decrease exponentially with increasing length of the fluctuation processes. This result is a retrospective justification for the neglect of fluctuations which lead beyond the range covered by the operators $F_{i, \alpha}$. Ground state (4) has charge properties which are size consistent and agree well with the results of Quantum Monte Carlo simulations.27

From Eq.(2) the $\mathrm{Cu} 2 p_{3 / 2}$ photoemission spectra are calculated using the Mori-Zwanzig projection technique 28 This method uses a set of operators $D_{\mu}$, the so-called dynamical variables. For these dynamical variables the following matrix equation holds

$$
\sum_{\gamma}\left(z \delta_{\mu \gamma}-\omega_{\mu \gamma}-\Sigma_{\mu \gamma}(z)\right) G_{\gamma \nu}(z)=\chi_{\mu \nu}
$$

where $z=\omega+i 0$, and where $\delta_{\mu \gamma}$ is the unity matrix. The correlation functions $G_{\gamma \nu}(z)$ are given by

$$
G_{\gamma \nu}(z)=\left\langle\Psi\left|D_{\gamma}^{\dagger} \frac{1}{z-\mathcal{L}} D_{\nu}\right| \Psi\right\rangle
$$

In Eq.(5) the susceptibility matrix $\chi_{\mu \nu}$, the frequency matrix $\omega_{\mu \gamma}$, and the self-energy matrix $\Sigma_{\mu \gamma}$ are defined by

$$
\begin{aligned}
\chi_{\mu \nu} & =\left\langle\Psi\left|D_{\mu}^{\dagger} D_{\nu}\right| \Psi\right\rangle \\
\omega_{\mu \gamma} & =\sum_{\eta}\left\langle\Psi\left|D_{\mu}^{\dagger} \mathcal{L} D_{\eta}\right| \Psi\right\rangle \chi_{\eta \gamma}^{-1}, \\
\Sigma_{\mu \gamma}(z) & =\sum_{\eta}\left\langle\Psi\left|D_{\mu}^{\dagger} \mathcal{L} Q \frac{1}{z-Q \mathcal{L} Q} Q \mathcal{L} D_{\eta}\right| \Psi\right\rangle \chi_{\eta \gamma}^{-1} .
\end{aligned}
$$

$Q$ is a projector on the subspace which is orthogonal to the space spanned by the dynamical variables $D_{\mu}$. It is defined by

$$
Q=1-\sum_{\mu \nu} D_{\mu}|\Psi\rangle \chi_{\mu \nu}^{-1}\langle\Psi| D_{\nu}^{\dagger}
$$

The set $\left\{D_{\mu}\right\}$ should contain the dynamical variable $D_{0 \xi}=c_{0 \xi}^{\dagger}$. Then, one of the correlation functions in
Eq.(7) is the hole-hole correlation function $G_{00}^{\xi}$ of Eq.(3). In this case, solving Eq.(6) for $G_{00}^{\xi}$ one obtains the spectral intensity $I(\omega)$ from Eq.(2). One possible approach to obtain an approximate solution of Eq.(6) is to make the set $\left\{D_{\mu}\right\}$ of dynamical variables sufficiently large so that the self-energies $\Sigma_{\mu \gamma}$ can be neglected. In this case an approximation for $I(\omega)$ results which can be systematically improved by further enlarging the set of dynamical variables until the results are converged. Due to the neglect of the self-energy matrix the calculated spectra show no broadening. Thus, a convolution with an artificial line width $\Gamma$ is necessary for a comparison with the experiment.

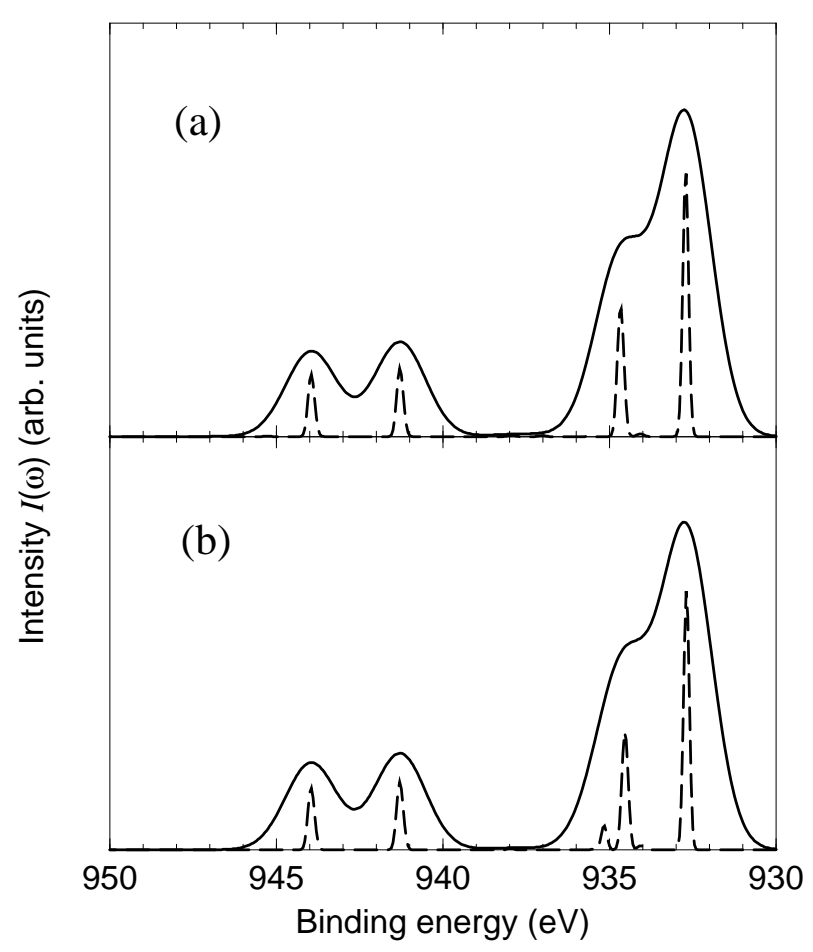

FIG. 1. Convergence of the projection technique. The calculated spectra for an infinite $\mathrm{CuO}_{3}$ chain are shown convoluted with a Gaussian function of width $\Gamma=1.8 \mathrm{eV}$ (solid lines) and $\Gamma=0.2 \mathrm{eV}$ (dashed lines). (a) and (b) show the result with 40 and 70 dynamical variables, respectively. The additional dynamical variables used in the second case describe far-reaching delocalization processes leading to the next-nearest neighbor plaquette. Their inclusion leads to a small redistribution of spectral weight around $935 \mathrm{eV}$ binding energy which is only visible for the smaller line width $0.2 \mathrm{eV}$.

Besides $D_{0 \xi}$ the chosen set of dynamical variables includes the operator

$$
D_{0 \xi}^{\prime}=\sum_{\sigma} d_{0,-\sigma}^{\dagger} d_{0 \sigma} c_{0, \xi+2 \sigma}^{\dagger}
$$

which describes the creation of a $\mathrm{Cu}$ core hole on site $i=0$ and a spin flip of the valence hole on the same site 
due to the exchange interaction with the core hole. Furthermore, we include the following dynamical variables

$$
\begin{aligned}
D_{\alpha \xi} & =F_{0, \alpha} c_{0 \xi}^{\dagger}, \\
D_{\alpha \xi}^{\prime} & =F_{0, \alpha} \sum_{\sigma} d_{0,-\sigma}^{\dagger} d_{0 \sigma} c_{0, \xi+2 \sigma}^{\dagger},
\end{aligned}
$$

for all fluctuation operators $F_{0, \alpha}$ used in the ground-state calculation. These dynamical variables describe the creation of a core hole and the subsequent delocalization of the valence hole from the same site, without $\left(D_{\alpha \xi}\right)$ and with spin flip $\left(D_{\alpha \xi}^{\prime}\right)$. In the case of a single $\mathrm{CuO}_{4}$ plaquette a set of 14 dynamical variables suffices for the exact solution. For a $\mathrm{CuO}_{2}$ plane 33 variables lead to a well-converged spectrum, while 40 variables are sufficient for a $\mathrm{CuO}_{3}$ chain.

In Fig. 1 the convergence of the results is exemplified for the case of $\mathrm{a} \mathrm{CuO}_{3}$ chain. Figure 1(a) shows the spectrum obtained with 40 dynamical variables while for the spectrum in Fig. 1(b) the set of dynamical variables is almost twice as large (70 variables). The inclusion of the additional variables leads to a small redistribution of spectral weight around $935 \mathrm{eV}$ binding energy. This change is only visible when a small line width $\Gamma$ is used for the convolution of the line spectrum.

\section{RESULTS}

A typical set of values for the parameters in model (1) has been obtained for $\mathrm{La}_{2} \mathrm{CuO}_{4}$ by band-structure calculation 29

$$
\begin{aligned}
\Delta & =3.5 \mathrm{eV}, U_{d}=8.8 \mathrm{eV}, \\
t_{p d} & =1.3 \mathrm{eV}, t_{p p}=0.65 \mathrm{eV} .
\end{aligned}
$$

For the comparison with the experimental spectra we start with this set and adapt some of the parameters to the specific systems which we investigate. The value of the exchange parameter $I_{d c}=-1.5 \mathrm{eV}$ and the line width $\Gamma=1.8 \mathrm{eV}$ of the Gaussian function to convolute the spectrum are obtained from a comparison of the exact solution for a single $\mathrm{CuO}_{4}$ plaquette with the experimental result for $\mathrm{Bi}_{2} \mathrm{CuO}_{4}$, see Fig. 2. From this comparison we furthermore determine the absolute energetical position of all calculated spectra. Thereby we allow for general shifts of $\pm 0.3 \mathrm{eV}$ which is the experimental accuracy of the absolute energy values. 2

To fit the experimental spectra, we vary $\Delta$ and $U_{d c}$ until the calculated satellite to main-peak intensity ratio $I_{s} / I_{m}$ coincides with the experimental one. It is not possible to use only $\Delta$ as fit parameter because the separation between the satellite and the main line is mainly determined by the difference $U_{d c}-\Delta$. However, we can reduce the number of free parameters by keeping this difference constant.

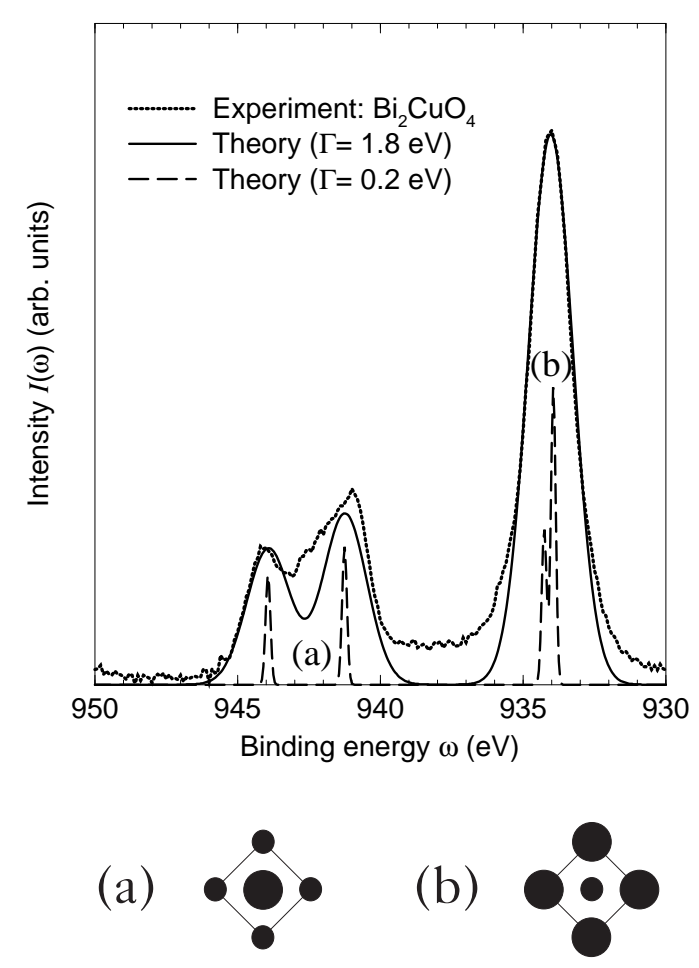

FIG. 2. $\mathrm{Bi}_{2} \mathrm{CuO}_{4}$ : Comparison of experimental data (dots) from Ref. 2 with the result of the projection technique for a single plaquette with $\Delta=3.5 \mathrm{eV}$. The theoretical spectra have been convoluted with a Gaussian function of width $\Gamma=1.8 \mathrm{eV}$ (solid line) and $\Gamma=0.2 \mathrm{eV}$ (dashed line). The valence hole delocalization in the final states is shown below. The core-hole site is the central $\mathrm{Cu}$ site. Large (small) dots symbolize a large (small) density of the valence hole originally located at the core-hole site. The final states (a) and (b) are associated to the corresponding lines in the spectrum.

For the spectra of $\mathrm{Bi}_{2} \mathrm{CuO}_{4}$ and $\mathrm{Sr}_{2} \mathrm{CuO}_{3}$ we keep $U_{d c}-\Delta=5 \mathrm{eV}$, while the smaller satellite-main line separation in the spectrum of $\mathrm{Sr}_{2} \mathrm{CuO}_{2} \mathrm{Cl}_{2}$ is taken into account by keeping $U_{d c}-\Delta=4.2 \mathrm{eV}$. In this way, we obtain a good fit of the $\mathrm{Bi}_{2} \mathrm{CuO}_{4}$ spectrum with $\Delta=3.5 \mathrm{eV}$, see Fig. 2, while the spectrum of $\mathrm{Sr}_{2} \mathrm{CuO}_{3}$ is well described by the result of the projection technique for a $\mathrm{CuO}_{3}$ chain with $\Delta=2.7 \mathrm{eV}$, see Fig 3 . These results have already been described elsewhere.26 Here, we therefore discuss only those aspects which are relevant for the influence of dimensionality and lattice geometry.

The lower parts of Figs. 2 and 3 show the delocalization properties of the final states which lead to the most important lines in the calculated spectra. The final state associated with the satellite lines, i.e. state (a) in Figs. 2 and 3 , is highly localized. Since the valence hole remains mainly on the core-hole site, the influence of geometry is negligible. This is consistent with the experimental observation that the satellite peaks depend much less on the geometry than the main lines. 

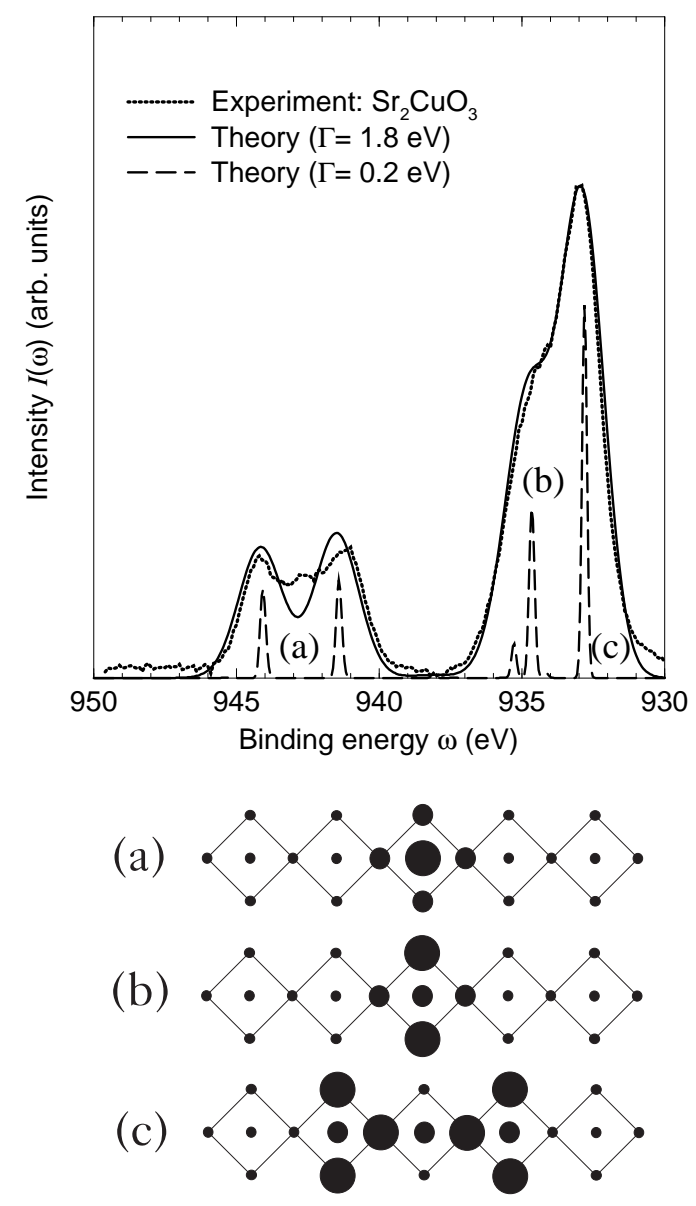

FIG. 3. $\mathrm{Sr}_{2} \mathrm{CuO}_{3}$ : Comparison of experimental data (dots) from Ref. 2 with the result of the projection technique for an infinite $\mathrm{CuO}_{3}$ chain with $\Delta=2.7 \mathrm{eV}$. The line width is $\Gamma=1.8 \mathrm{eV}$ (solid line) and $\Gamma=0.2 \mathrm{eV}$ (dashed line). The valence hole delocalization in the final states is shown below. The core-hole site is the $\mathrm{Cu}$ site in the central plaquette. Large (small) dots symbolize a large (small) density of the valence hole originally located at the core-hole site. The final states (a), (b) and (c) are associated to the corresponding lines in the spectrum.

Final state (b) which causes the main line in the $\mathrm{CuO}_{4}$ plaquette spectrum (Fig. 2) leads to a shoulder in the main line of the $\mathrm{CuO}_{3}$ chain spectrum (Fig. 3) and is also rather localized, with most of the valence hole density concentrated on the $\mathrm{O}$ sites surrounding the core-hole site. However, in the case of the $\mathrm{CuO}_{3}$ chain the hole density at the $\mathrm{O}$ sites in chain direction is smaller than at the $\mathrm{O}$ sites perpendicular to it. A comparable effect has been observed in exact diagonalization studies. $19.18,30$ The most important effect of the network geometry is the emergence of peak (c) in the $\mathrm{CuO}_{3}$ chain spectrum, see Fig. 3. This peak, which dominates the main line, is associated with a delocalization of the valence hole to the neighboring plaquettes which may heinterpreted as the formation of a Zhang-Rice singlet.18.15

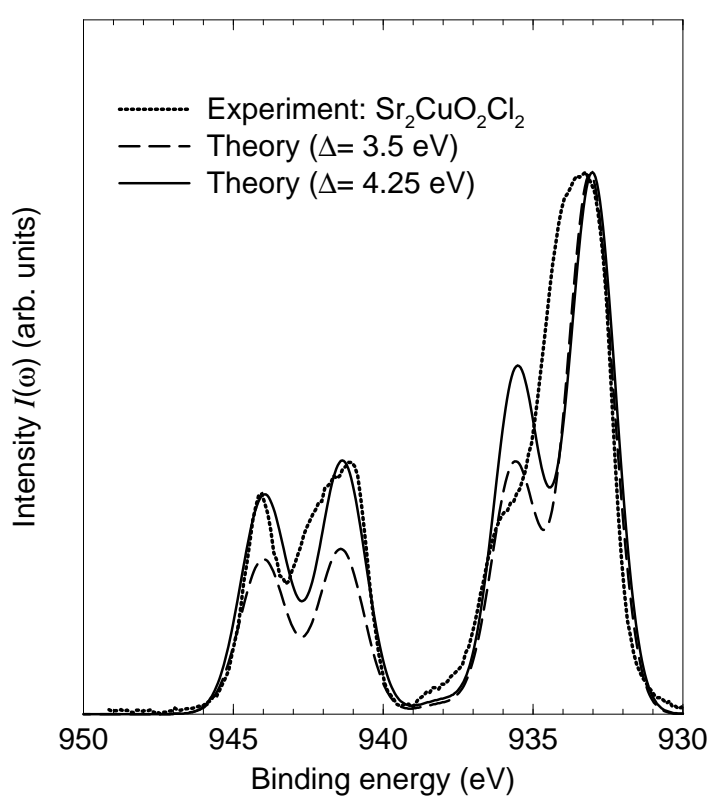

FIG. 4. $\mathrm{Sr}_{2} \mathrm{CuO}_{2} \mathrm{Cl}_{2}$ : Comparison of experimental data (dots) from Ref. 2 with the result of the projection technique for an infinite $\mathrm{CuO}_{2}$ plane. $\Delta$ is used as a fit parameter: $\Delta=3.5 \mathrm{eV}$ (dashed line) and $\Delta=4.25 \mathrm{eV}$ (solid line), all other parameter values are those of set (12). The line width is in both cases $\Gamma=1.8 \mathrm{eV}$. For $\Delta=4.25 \mathrm{eV}$ the intensity ratio $I_{s} / I_{m}$ between satellite and main line is equal to the experimental one, but the form of the main line is not reproduced correctly.

In the case of the $\mathrm{CuO}_{2}$ plane it turns out that a fit using $\Delta$ as described above does not lead to a satisfying agreement with the spectrum of $\mathrm{Sr}_{2} \mathrm{CuO}_{2} \mathrm{Cl}_{2}$, see Fig. 4 . For the standard value $\Delta=3.5 \mathrm{eV}$ (dashed line in Fig. 4) the calculated ratio $I_{s} / I_{m}=0.4$ is too small compared to the experimental value $I_{s} / I_{m}=0.52$. With increasing $\Delta$ (i.e. with decreasing charge fluctuations) the relative intensity of both the satellite and the shoulder structure around $935 \mathrm{eV}$ binding energy increases. For $\Delta=4.25 \mathrm{eV}$ (solid line in Fig. 4) the calculated ratio $I_{s} / I_{m}$ is equal to the experimental one. However, the form of the main line is not reproduced correctly. The intensity of the shoulder structure is overestimated while a line seems to be missing around $934 \mathrm{eV}$ binding energy.

In order to obtain a better fit we have varied $t_{p d}$ and $U_{d c}$ instead of $\Delta$ and $U_{d c}$. As shown in Fig. 5 this leads to a better agreement with the spectrum of $\mathrm{Sr}_{2} \mathrm{CuO}_{2} \mathrm{Cl}_{2}$. Keeping $\Delta=3.5 \mathrm{eV}$, the experimental ratio $I_{s} / I_{m}=0.52$ is reproduced for $t_{p d}=1.15 \mathrm{eV}$ and $U_{d c}=8.1 \mathrm{eV}$. The delocalization properties of the most important final states are shown in the lower part of Fig.5. As opposed to the zero- and one-dimensional cases shown in Figs. 2 and 3, the valence hole from the core-hole site may now delocalize in two dimensions. Nevertheless the final states obtained for a $\mathrm{CuO}_{2}$ plane are rather similar to those of the lower dimensional systems. For state (a) 
this is easy to explain by the local nature of the satellite peak. The fact that the delocalization in states (b) and (c) is not significantly larger in two dimensions than in one dimension is, on the other hand, somewhat surprising. Key element in the explanation of this effect are the four $\mathrm{Cu}$ sites which are the diagonal nearest neighbors of the core-hole site (cf. the lower part of Fig. 5). Due to antiferromagnetic correlations these diagonal $\mathrm{Cu}$ sites are predominantly occupied by valence holes which have the same spin direction as the valence hole on the core-hole site. Therefore, due to the Pauli principle the holes from the diagonal $\mathrm{Cu}$ sites suppress fluctuations of the valence hole from the core-hole site. The increase of charge fluctuations due to the higher dimensionality is largely compensated by this suppression. An analogous effect has already been found to be important for the ground-state charge properties of Hamiltonian (11) 27

It is interesting to compare the results of the projection technique with exact diagonalization calculations (without multiplet splitting) of a $\mathrm{Cu}_{5} \mathrm{O}_{16}$ cluster from Refs. 2.17. This cluster contains five plaquettes in a cross-like configuration where the central $\mathrm{Cu}$ site is the core-hole site. Notice that the $\mathrm{Cu}_{5} \mathrm{O}_{16}$ system does not contain the diagonal $\mathrm{Cu}$ sites which, as discussed above, suppress fluctuations from the central $\mathrm{Cu}$ site. Therefore one expects this system to display features of artificially strong delocalization like, e.g., a reduced ratio $I_{s} / I_{m}$. In fact, while the diagonalization shows a similar shoulder structure as the projection technique, cf. Fig. 4, the intensity of this shoulder, its separation from the lowestenergy line as well as the ratio $I_{s} / I_{m}$ are smaller.

The slightly reduced value $t_{p d}=1.15 \mathrm{eV}$ for the $\mathrm{Cu}-\mathrm{O}$ hopping strength which leads to the spectrum shown in Fig. 5 may be explained by the larger $\mathrm{Cu}-\mathrm{O}$ distance in $\mathrm{Sr}_{2} \mathrm{CuO}_{2} \mathrm{Cl}_{2}$ compared to $\mathrm{La}_{2} \mathrm{CuO}_{4} .5$ Nevertheless, the agreement of the theoretical result with the experimental spectrum is still not satisfactory. The calculated main line is dominated by two features, and at least one excitation seems to be missing in the region around $934 \mathrm{eV}$ binding energy. Notice, that in the theoretical main line of $\mathrm{Sr}_{2} \mathrm{CuO}_{2} \mathrm{Cl}_{2}$ from Ref. 22 an excitation is missing as well.

These results suggest the conclusion that Hamiltonian (11) still does not include all degrees of freedom which are necessary for a detailed description of the main line in the $\mathrm{Cu} 2 \mathrm{p}_{3 / 2}$ spectrum of $\mathrm{Sr}_{2} \mathrm{CuO}_{2} \mathrm{Cl}_{2}$. Since all effects of the $\mathrm{Cu}-\mathrm{O}$ network dimensionality are already included in model (11) we expect the missing third main-line feature to be a material-specific effect. Orbitals which are not yet taken into account are, for example, non-planar orbitals in the $\mathrm{CuO}_{2}$ system, like the $\mathrm{Cu} 3 d_{z^{2}-r^{2}}$ orbital. However, in view of the good agreement with the experiments shown in Figs. 2 and 3, these orbitals do not seem necessary for the description of $\mathrm{Bi}_{2} \mathrm{CuO}_{4}$ and $\mathrm{Sr}_{2} \mathrm{CuO}_{3}$.
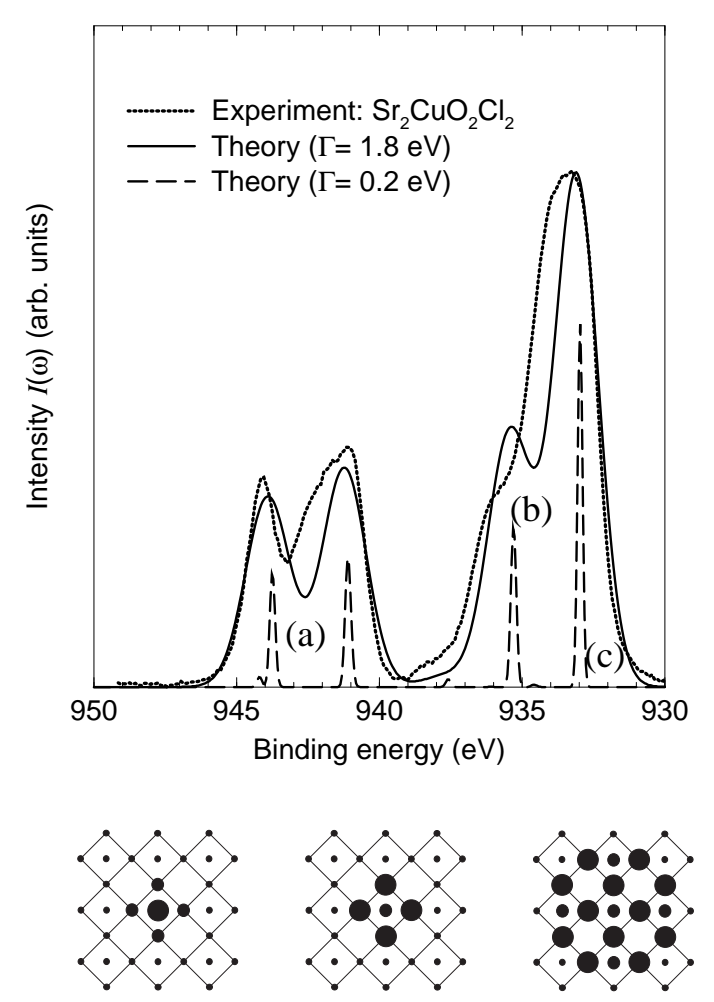

(a)

(b)

(c)

FIG. 5. $\mathrm{Sr}_{2} \mathrm{CuO}_{2} \mathrm{Cl}_{2}$ : Comparison of experimental data (dots) from Ref. $\mathrm{O}$ with the result of the projection technique for an infinite $\mathrm{CuO}_{2}$ plane. $t_{p d}$ is used as a fit parameter. $t_{p d}=1.15 \mathrm{eV}$ and $U_{d c}=8.1 \mathrm{eV}$. The line width is $\Gamma=1.8 \mathrm{eV}$ (solid line) and $\Gamma=0.2 \mathrm{eV}$ (dashed line). The valence hole delocalization in the final states is shown below. The core-hole site is the $\mathrm{Cu}$ site in the central plaquette. Large (medium, small) dots symbolize a large (medium, small) density of the valence hole originally located at the core-hole site. The final states (a), (b) and (c) are associated to the corresponding lines in the spectrum.

It may also be possible that sites which do not belong to the $\mathrm{CuO}_{2}$ plane (like the $\mathrm{Cl}$ apex site) contribute to the screening in $\mathrm{Sr}_{2} \mathrm{CuO}_{2} \mathrm{Cl}_{2}$. Notice that the $\mathrm{Cu}$ $2 p$ main line in copper oxides without apex-oxygen site $\left(\mathrm{CuO}, \mathrm{Nd}_{2} \mathrm{CuO}_{4}\right)$ is narrower than in compounds with one apex oxygen $\left(\mathrm{Bi}_{2} \mathrm{Sr}_{2} \mathrm{CaCH}_{2} \mathrm{O}_{8}\right)$ or two apex oxygens $\left(\mathrm{La}_{2} \mathrm{CuO}_{4}\right)$ per copper site 31 However, both the large spatial distance between the $\mathrm{Cl}$ and the $\mathrm{CuO}_{2}$ plane and the large energy difference between the $\mathrm{Cl}-3 p$ and the $\mathrm{Cu}-3 d-\mathrm{O}-2 p$ line in the valence photoelectron spectrum suggest that screening from $\mathrm{Cl}$ sites should be small. Another possible candidate for the explanation of the missing third main-line feature are non-bonding oxygen $2 p$ orbitals. Recently, a feature in the optical spectrum of $\mathrm{Sr}_{2} \mathrm{CuO}_{2} \mathrm{Cl}_{2}$ has been attributed to these orbitals. 32 


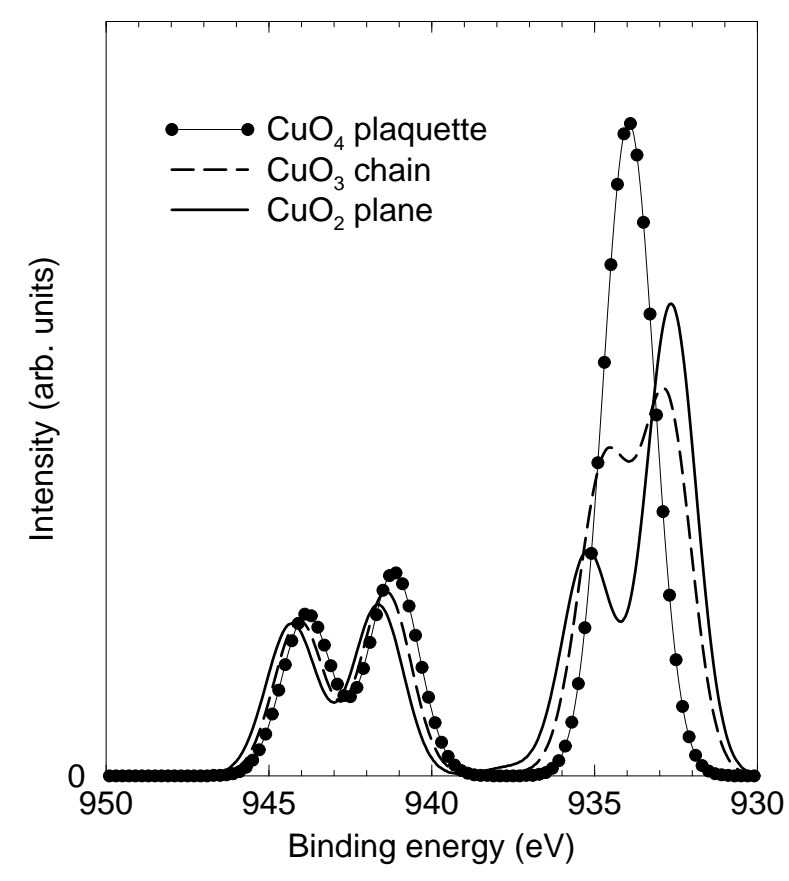

FIG. 6. Effects of $\mathrm{Cu}-\mathrm{O}$ network dimensionality. Since the spectra of the three different $\mathrm{Cu}-\mathrm{O}$ networks are shown using the same parameter set (12) in all cases, all differences are exclusively due to dimensionality effects. The line width is $\Gamma=1.8 \mathrm{eV}$, while $U_{d c}=8.5 \mathrm{eV}$, and $I_{d c}=-1.5 \mathrm{eV}$. The satellite to main line intensity ratio $I_{s} / I_{m}$ is 0.56 for the $\mathrm{CuO}_{4}$ plaquette, 0.50 for the $\mathrm{CuO}_{3}$ chain, and 0.49 for the $\mathrm{CuO}_{2}$ plane.

The influence of $\mathrm{Cu}-\mathrm{O}$ network dimensionality on the $\mathrm{Cu} 2 p_{3 / 2}$ spectra is illustrated in Fig. 6 where we show results of the projection technique for zero-, one- and twodimensional $\mathrm{Cu}-\mathrm{O}$ networks. Since the same parameter set (12) has been used for all geometries, all changes in the spectra are exclusively due to dimensionality effects. The most important effect in the spectra is that for the one- and two-dimensional system an additional excitation appears at lower binding energies. As discussed above the final states of this excitation are delocalized and rather similar for both the one- and the two-dimensional structures, see states (c) in Figs. 3 and 5. Overall, there is only a quantitative change from one to two dimensions, in contrast to the qualitative change observed between zero and higher dimensions. This conclusion is in principal agreement with results of exact diagonalization calculations. 17 The peak around $934 \mathrm{eV}$ binding energy which dominates the main line in the case of zero dimensions becomes a shoulder structure which decreases in intensity and shifts towards higher binding energies as the dimensionality increases. Nevertheless, the final state associated with this peak preserves its main properties (a large valence-hole density at the $\mathrm{O}$ sites around the core-hole site) with changing dimensionality, see states (a) in Figs. 2, 3, and 5. As the dimensionality increases the delocalization in the $\mathrm{Cu}-\mathrm{O}$ network increases. Therefore one observes a monotonic decrease in the ratio $I_{s} / I_{m}$ for increasing dimensions. Since this trend is not observed experimentally, the actual value of $I_{s} / I_{m}$ has to depend mainly on material-specific properties. In our calculation these properties are reflected by the values of the model parameters, cf. Figs. 2 to 5 .

\section{SUMMARY}

Summing up, we have calculated the $\mathrm{Cu} 2 p_{3 / 2}$ corelevel spectra of zero- one- and two-dimensional $\mathrm{Cu}-\mathrm{O}$ networks using a model Hamiltonian which describes exchange splitting and delocalization within one framework. The spectral intensity has been obtained using the Mori-Zwanzig projection technique. This method leads to the exact solution for a single $\mathrm{CuO}_{4}$ plaquette, and we observe excellent convergence in the case of an infinite $\mathrm{CuO}_{3}$ chain and an infinite $\mathrm{CuO}_{2}$ plane. The delocalization properties of the final states obtained in the calculation are easy to interpret. The results have been compared to experimental spectra by using either $\Delta$ or $t_{p d}$ as a fit parameter. While there is a good agreement between theory and experiment in the case of $\mathrm{Bi}_{2} \mathrm{CuO}_{4}$ and $\mathrm{Sr}_{2} \mathrm{CuO}_{3}$, one excitation seems to be missing when compared with the spectrum of $\mathrm{Sr}_{2} \mathrm{CuO}_{2} \mathrm{Cl}_{2}$. An analysis of the influence of dimensionality effects, as compared to effects due to material-specific properties, indicates that this missing feature may be due to orbitals which are not contained in the model. It is also found that dimensionality plays a minor role for the satellite-main line intensity ratio $I_{s} / I_{m}$ which is mainly determined by the values of the model parameters (especially the value of $\Delta$ ).

\section{ACKNOWLEDGMENTS}

Discussions with J. Fink, M. S. Golden, A. Goldoni, R. E. Hetzel, F. Parmigiani, and L. Sangaletti are gratefully acknowledged. This work is supported by DFG through the research program of the SFB 463, Dresden.

${ }^{1}$ T. Böske, O. Knauff, R. Neudert, M. Kielwein, M. Knupfer, M. S. Golden, J. Fink, H. Eisaki, S. Uchida, K. Okada, and A. Kotani, Phys. Rev. B 56, 3438 (1997).

${ }^{2}$ T. Böske, K. Maiti, O. Knauff, K. Ruck, M. S. Golden, G. Krabbes, J. Fink, T. Osafune, N. Motoyama, H. Eisaki, and S. Uchida, Phys. Rev. B 57, 138 (1998).

${ }^{3}$ G. van der Laan, C. Westra, C. Haas, and G. A. Sawatzky, Phys. Rev. B 23, 4369 (1981).

${ }^{4}$ A. Fujimori, E. Takayama-Muromachi, Y. Uchida, and B. Okai, Phys. Rev. B 35, 8814 (1987). 
${ }^{5}$ Z.-X. Shen, J. W. Allen, J. J. Yeh, J.-S. Kang, W. Ellis, W. Spicer, I. Lindau, M. B. Maple, Y. D. Dalichaouch, M. S. Torikachvili, J. Z. Sun, and T. H. Geballe, Phys. Rev. B 36, 8414 (1987).

${ }^{6}$ J. Ghijsen, L. H. Tjeng, J. van Elp, H. Eskes, J. Westerink, G. A. Sawatzky, and M. T. Czyzyk, Phys. Rev. B 38, 11322 (1988).

${ }^{7}$ A. Kotani and Toyozawa, J. Phys. Soc. Japan 35, 1073, 1082 (1973).

${ }^{8}$ S. Larsson and M. Braga, Chem. Phys. Lett. 48, 596 (1977).

${ }^{9}$ K. Okada and A. Kotani, J. Phys. Soc. Japan 58, 2578 (1989).

10 A. Goldoni, U. del Pennino, F. Parmigiani, L. Sangaletti, and A. Revcolevschi, Phys. Rev. B 50, 10435 (1994).

${ }^{11}$ M. A. van Veenendaal and G. A. Sawatzky, Phys. Rev. B 49, 3473 (1994).

12 S. Tanaka and T. Jo, Physica B 237-238, 385 (1997).

${ }^{13}$ F. Parmigiani, Z. X. Shen, D. B. Mitzi, I. Lindau, W. E. Spicer, and A. Kapitulnik, Phys. Rev. B 43, 3085 (1991).

14 M. A. van Veenendaal and G. A. Sawatzky, Phys. Rev. Lett. 70, 2459 (1993)

${ }^{15}$ M. A. Veenendaal, H. Eskes, and G. A. Sawatzky, Phys. Rev. B 47, 11462 (1993).

${ }^{16}$ F. C. Zhang and T. M. Rice, Phys. Rev. B 37, 3759 (1988).

${ }^{17}$ K. Okada and A. Kotani, J. Electron. Spectrosc. Relat. Phenom. 86, 119 (1997).

${ }^{18}$ K. Okada and A. Kotani, Phys. Rev. B 52, 4794 (1995).

${ }^{19}$ K. Okada, A. Kotani, K. Maiti, and D. D. Sarma, J. Phys. Soc. Japan 65, 1844 (1996).

${ }^{20}$ O. Gunnarsson and K. Schönhammer, Phys. Rev. Lett. 50,
604 (1983).

${ }^{21}$ O. Gunnarsson and K. Schönhammer, Phys. Rev. B 28, 4315 (1983).

${ }^{22}$ K. Karlsson, O. Gunnarsson, and O. Jepsen, Phys. Rev. Lett. 82, 3528 (1999).

${ }^{23}$ E. W. Ong, G. H. Kwei, R. A. Robinson, B. L. Ramakrishna, R. B. Von Dreele, Phys. Rev. B 42, 4255 (1990).

${ }^{24}$ T. Ami, M. K. Crawford, R. L. Harlow, Z. R. Wang, D. C. Johnston, Q. Huang, and R. W. Erwin, Phys. Rev. B 51, 5994 (1995)

${ }^{25}$ L. L. Miller, X. L. Wang, S. X. Wang, C. Stassis, D.C. Johnston, J. Faber Jr., and C.-K. Loong, Phys. Rev. B 41, 1921 (1990)

${ }^{26}$ C. Waidacher, J. Richter, and K. W. Becker, Europhys. Lett. 47, 77 (1999).

${ }^{27}$ C. Waidacher, J. Richter, R. E. Hetzel, and K. W. Becker, Phys. Rev. B 60, 2255 (1999).

${ }^{28}$ H. Mori, Prog. Theor. Phys. 33, 423 (1965); R. Zwanzig, in Lectures in Theoretical Physics (Interscience, New York, 1961), Vol. 3

${ }^{29}$ A. K. McMahan, R. M. Martin, and S. Satpathy, Phys. Rev. B 38, 6650 (1988); M. S. Hybertsen, M. Schlüter, and N. E. Christiansen, Phys. Rev. B 39, 9028 (1989).

${ }^{30}$ K. Okada and A. Kotani, J. Electron. Spectrosc. Relat. Phenom. 88-91, 255 (1998).

${ }^{31}$ F. Parmigiani, L. E. Depero, T. Minerva, and J. B. Torrance, J. Electron. Spectrosc. Relat. Phenom. 58, 315 (1992).

${ }^{32}$ H. S. Choi, Y. S. Lee, T. W. Noh, E. J. Choi, Y. Bang, and Y. J. Kim, Phys. Rev. B 60, 4646 (1999). 